\title{
Miliary Tuberculosis with Hypercalcemia, and a False Abdominal Aortic Aneurysm, but No Pulmonary Findings
}

\author{
Yoko Uchiyama-Tanaka ${ }^{1}$ and Yasukiyo Mori ${ }^{2}$
}

\begin{abstract}
A 69-year-old man with a 10-year history of diabetes mellitus and 1-year history of steroid treatment for nonspecific interstitial pneumonia presented with a nightly fever of over $39^{\circ} \mathrm{C}$ after surgery for a false abdominal aortic aneurysm. Hypercalcemia was detected, despite acute renal dysfunction. There was no laboratory evidence of collagen disease or infection including tuberculosis. Polymerase chain reaction (PCR)-based amplification of DNA from a bone marrow biopsy specimen revealed Mycobacterium tuberculosis. Antituberculous chemotherapy was initiated. Early bone marrow biopsy and the use of new techniques such as PCR can avoid delay in initiating the proper treatment for compromised patients.
\end{abstract}

Key words: miliary tuberculosis, aneurysm, hypercalcemia

(DOI: 10.2169/internalmedicine.45.1740)

\section{Introduction}

After a steady decline in the incidence of tuberculosis (TB), an increase has recently been noted worldwide, according to the World Health Organization (1). Host resistance to TB is mediated by cellular immunity, and because this is impaired by steroid therapy and by diabetes mellitus (DM) (2-4), patients under these conditions are susceptible to $\mathrm{TB}(2,5,6)$. TB in such patients is characterized by increasing extrapulmonary involvement and miliary spread $(2$, $3,6-8$ ). The term miliary TB, first used to describe resemblance of the pathologic lesions to millet seeds, is now used for all forms of progressive disseminated hematogenous TB regardless of the pathologic picture. The sputum smear is positive in about $30 \%$ of patients $(7,8)$. Results of sputum culture are positive in a higher percentage of patients, but therapy cannot be delayed to allow time for culture. Mortality associated with miliary TB remains high; despite the availability of effective therapies, reported mortality rates are $16-28 \%(7,8)$. Early diagnosis is important for patient survival. Cryptic miliary TB is a clinical term introduced to describe an atypical presentation, usually in older patients, in which the usual markers of the disease are lacking. In such cases, the diagnosis is difficult because of normal chest roentgenograms, negative tuberculin tests, and often a confounding underlying illness to which symptoms are mistakenly attributed (9). In most cases of cryptic miliary TB, chronic foci of organ TB are associated with intermittent, nonprogressive seeding via the bloodstream. However, a compromised state may become continuous and case progressive hematogenous TB, a sign of progressive generalized TB occurring long after the primary infection (10). This type of miliary TB is often diagnosed at autopsy. According to one study, cryptic miliary TB is often clinically silent, deriving from renal, genitourinary, or osseous foci or deep lymph nodes in the mediastinum or abdomen (10).

The clinical manifestations of extrapulmonary TB are usually nonspecific and obscure. Aneurysm is an infrequent complication of TB $(11,12)$. Parkhurst et al reported the incidence of TB aneurysm to be only $0.3 \%$ (13). Most of these aneurysms are identified at autopsy; however, some recent reports describe successful surgical treatment of $\mathrm{TB}$ aneurysm.

Here, we report a rare case of miliary TB without pulmonary features that was diagnosed by polymerase chain reaction (PCR)-based amplification of DNA from a bone marrow sample in a patient with acute renal failure, hypercalce-

\footnotetext{
${ }^{1}$ The Renal Center, Takeda Hospital, Kyoto and ${ }^{2}$ Department of Medicine, Division of Cardiology and Nephrology, Kyoto Prefectural University of Medicine, Kyoto

Received for publication January 27, 2006; Accepted for publication August 21, 2006

Correspondence to Dr. Yoko Uchiyama-Tanaka, The Renal Center, Takeda Hospital, Nishinotouinhigashiiri, Shiokojidori, Shimokyo-ku, Kyoto $600-8558$
} 

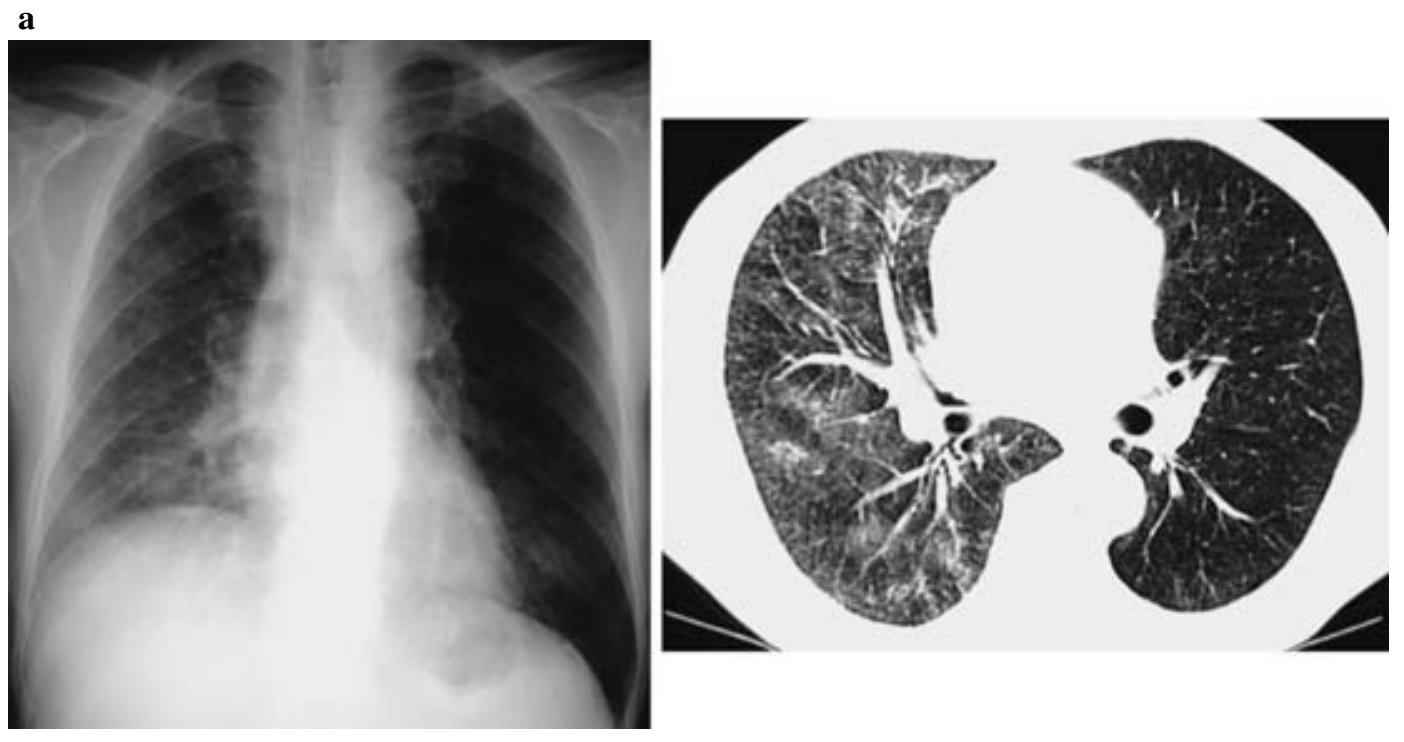

b



Figure 1. a: Chest CT and $\mathrm{X}$ ray on the basis of which nonspecific interstitial pneumonia was diagnosed in June 2004 at a prior hospital. At that time, this patient refused to undergo any biopsy examinations, so this diagnosis was made by exclusion. b: Chest CT and X ray upon admission at our hospital in June 2005. There are no particular findings.

mia, and leukopenia after surgical treatment of a false abdominal aortic aneurysm.

\section{Case Report}

An abdominal aortic aneurysm was diagnosed in a 69year-old man on the basis of abdominal pain and abdominal computed tomography (CT) findings. He had been undergoing steroid therapy for nonspecific interstitial pneumonia for 1 year since June 2004 (Fig. 1a) and was taking prednisolone at $5 \mathrm{mg}$ per day at the time of admission. The patient also had DM that had been untreated for 10 years which was complicated by nephropathy. The patient was admitted to our hospital and underwent surgery in June, 2005. The aortic wall structure was destroyed and showed necrosis.
These findings suggested infectious aneurysm, but the etiology could not be determined. The aneurysm did not involve the renal arteries. The patient underwent enhanced CT study (Fig. 2) and coronary cardioangiography. Laboratory tests showed that his serum creatinine ( $\mathrm{s}-\mathrm{Cr}$ ) concentration was $1.3 \mathrm{mg} / \mathrm{dl}$ in March 2005 and $4.5 \mathrm{mg} / \mathrm{dl}$ upon admission in early June, 2005, and that it increased gradually to $6.5 \mathrm{mg} /$ dl by June 25, 2005, 2 weeks after the surgery. The patient was referred to our center for examinations related to renal dysfunction to determine whether dialysis was indicated. At that time, he began to show a fever of more than $39^{\circ} \mathrm{C}$ every night.

Upon admission to our center, the patient's blood pressure was $100 / 60 \mathrm{mmHg}$, his pulse was 90 beats/min, and his body temperature was $36^{\circ} \mathrm{C}$ in the morning and $39^{\circ} \mathrm{C}$ at 


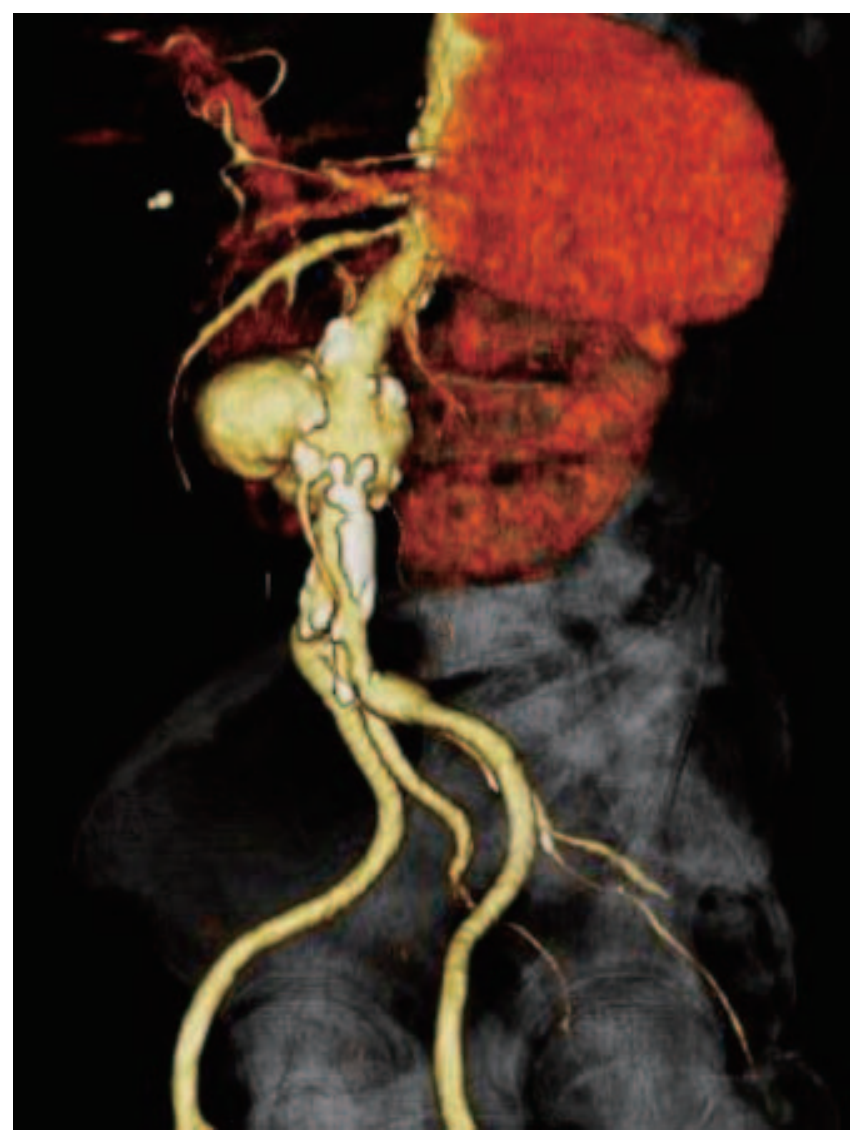

Figure 2. Three-dimensional angiographic image of the abdominal aortic aneurysm at admission.

night. Complete physical examination, including the lungs, heart, and abdomen, revealed no abnormality except numbness of the lower limbs.

The laboratory findings were as follows: creatinine clearance, $6.5 \mathrm{ml} / \mathrm{min}$; erythrocytes, $422 \times 10^{4} / \mu \mathrm{l}$; hemoglobin,

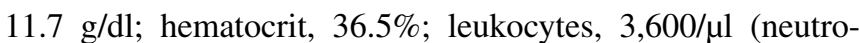
phils $80 \%$, lymphocytes $14 \%$ ); and platelets, $18.6 \times 10^{4} / \mu \mathrm{l}$. Blood chemistry tests yielded the following: blood urea nitrogen, $76.5 \mathrm{mg} / \mathrm{dl}$; s-Cr, $6.5 \mathrm{mg} / \mathrm{dl}$; total protein, $5.5 \mathrm{~g} / \mathrm{dl}$; albumin, $2.7 \mathrm{~g} / \mathrm{dl}$; aspartate aminotransferase, $18 \mathrm{U} / \mathrm{l}$; alanine aminotransferase, $14 \mathrm{U} / \mathrm{l}$; calcium, $11 \mathrm{mg} / \mathrm{dl}$ (12.3 mg after correction for albumin); phosphorus, $4.2 \mathrm{mg} / \mathrm{dl}$; fasting blood glucose, $95 \mathrm{mg} / \mathrm{dl}$; hemoglobin A1c, 6.1\%; C-reactive protein (CRP) $8.6 \mathrm{mg} / \mathrm{dl}$. There were no laboratory findings suggestive of collagen disease. Results of virologic tests were negative for hepatitis $\mathrm{C}$ and $\mathrm{B}$, cytomegalovirus, herpes virus, Epstein Barr virus, and human immunodeficiency virus. There was no evidence of systemic fungal infection. Blood, sputum, stool, and urine cultures were negative for all pathogens including Mycobacterium tuberculosis. Chest X-ray examination, CT (Fig. 1b), and electrocardiography showed no abnormalities.

A granulomatous disorder such as sarcoidosis, TB, or malignancy was suspected because of the hypercalcemia and depressed intact parathyroid hormone $(2 \mathrm{pg} / \mathrm{ml})$ and normal 1,2 dihydroxy vitamin $\mathrm{D}(36 \mathrm{pg} / \mathrm{dl})$ concentrations. There
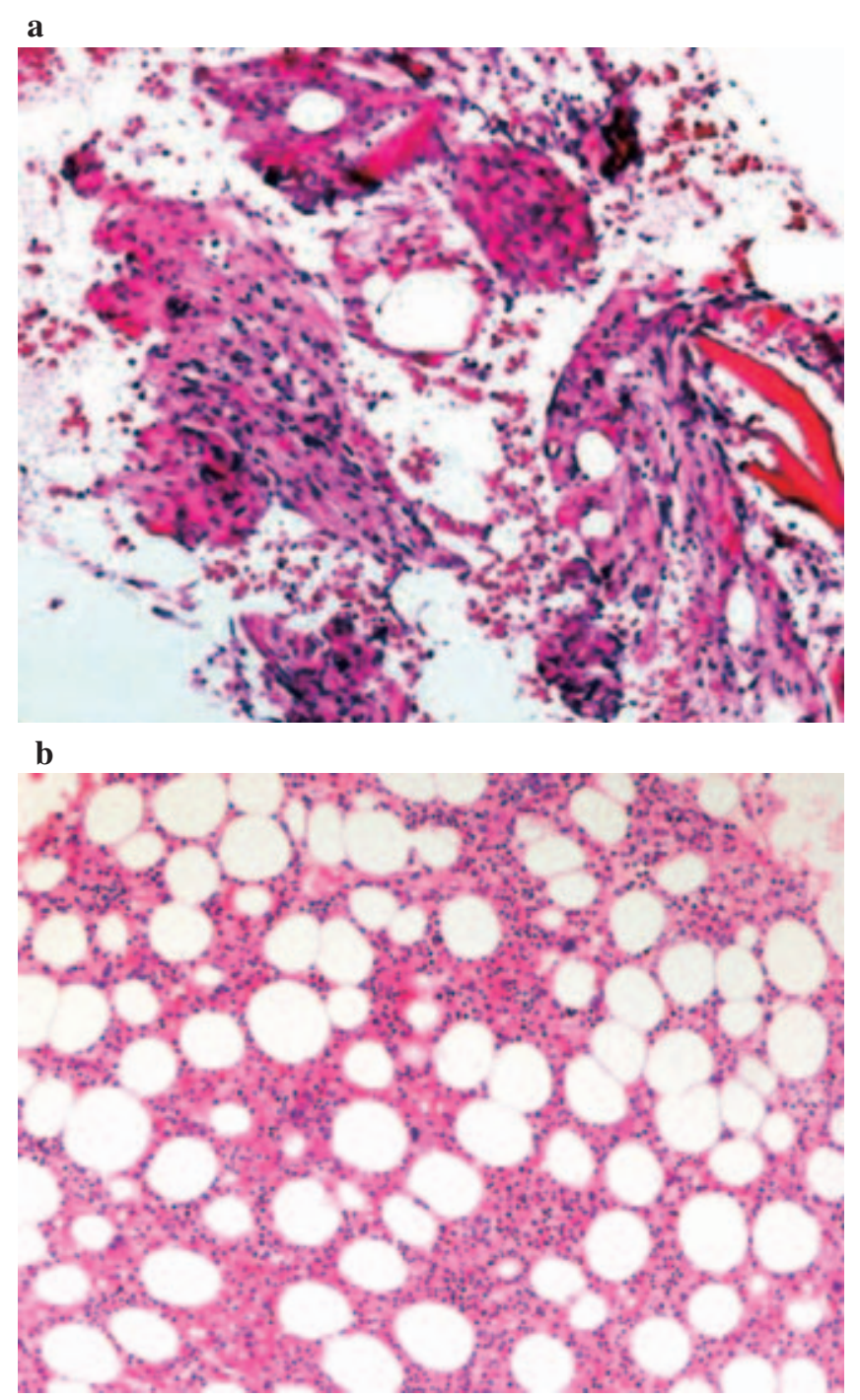

Figure 3. a: Light microscopy showed noncaseous granuloma in bone marrow in July 2005 (periodic acid-Schiff staining, $\times 200)$. b: Light microscopy showed no abnormality in bone marrow in May 2006 (periodic acid-Schiff staining, $\times 200)$. There is no granuloma.

was no Bence-Jones protein in the urine, monoclonal antibody in serum, or punched-out lesion of the skull. Gallium scintigraphy and bone scintigraphy showed no specific accumulation, and there were no abnormalities on abdominal ultrasonography images.

Sarcoidosis was strongly suspected because of the fever of unknown origin, negative tuberculin test, and elevated lysozyme $(29.8 \mu \mathrm{g} / \mathrm{ml})$ and soluble interleukin 2 receptor $(3413$ $\mathrm{U} / \mathrm{ml}$ ) concentrations. Leukopenia and anemia were detected [erythrocytes, $283 \times 10^{4} / \mu \mathrm{l}$; hemoglobin, $7.7 \mathrm{~g} / \mathrm{dl}$; hematocrit, $23.7 \%$; leukocytes, $1,700 / \mu$ l (neutrophils $79 \%$, lymphocytes $17 \%)]$. Because sarcoidosis with bone marrow involvement was suspected, the patient was treated with $30 \mathrm{mg} /$ day of oral prednisolone after steroid pulse therapy (methylprednisolone sodium succinate of $500 \mathrm{mg}$ per day for 3 days) beginning on July 21 . After pulse therapy, the patient's nightly 


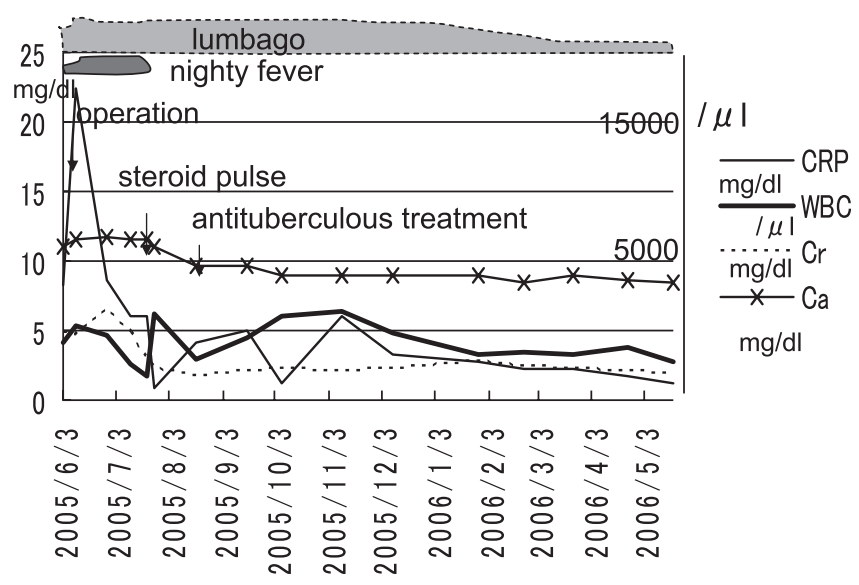

Figure 4. The course of the main laboratory findings and clinical symptoms. $\mathrm{Cr}$, creatinine; WBC, white blood cell and leukocyte counts; Alb, albumin; Ca, calcium after correction for albumin.

fever disappeared, his CRP concentration decreased, and he recovered from the leukopenia. However, the CRP concentration never returned to normal, and after 2 weeks, the leukopenia recurred. The patient finally agreed to undergo bone marrow aspiration and biopsy, and both procedures were performed on August 17. Bone marrow aspiration failed (dry tap), but marrow biopsy and culture were performed. The smear was positive for acid-fast bacteria, and polymerase chain reaction (PCR) for TB DNA (COBAS AMPLICOR $®)$ in bone marrow was positive 3 days after the biopsy. Therefore, the patient was treated with antituberculous chemotherapy (rifampicin at $450 \mathrm{mg} /$ day, isoniazid at $300 \mathrm{mg} /$ day, levofloxacin at $200 \mathrm{mg} /$ day). The bone marrow sample contained noncaseous granuloma (Fig. 3a). The patient's renal function improved before the start of steroid therapy, and tubulointerstitial nephritis was diagnosed pathologically with renal biopsy. On the basis of our findings, we suspected that the renal lesion was tubulointerstitial nephritis, induced by nonsteroidal anti-inflammatory drugs given for lumbago and the ionized drug used to obtain images, and related to the diabetic nephropathy. We concluded that the long-term steroid therapy and DM induced miliary $\mathrm{TB}$, which was complicated by a false aortic aneurysm. The patient's glomerular filtration rate was still $25 \mathrm{ml} / \mathrm{min}$. He had no symptoms after steroid therapy and antituberculous treatment except for the lumbago, which was controlled by medication. Corticosteroid therapy was tapered, and the patient was discharged in September, 2005.

Although liver dysfunction necessitated reduction of rifampicin to $300 \mathrm{mg} / \mathrm{day}$, nightly fever, hypercalcemia, and leukopenia did not recur, and the patient recovered from lumbago 6 months after the initiation of antituberculous treatment. However, the patient's CRP concentration remained elevated (Fig. 4), therefore bone marrow aspiration and biopsy were performed again in May, 2006. The smear was negative for acid-fast bacteria, and PCR for TB DNA was negative. The bone marrow sample showed no abnormality (Fig. 3b). Therefore, antituberculous treatment was stopped on May 18, 2006, 9 months after it was started.

\section{Discussion}

TB remains a serious health problem worldwide; according to recent reports, the annual incidence is increasing slightly. Patients with DM and those taking immunosuppressive medications are susceptible to $\mathrm{TB}$ and other forms of mycobacteriosis. TB infection is perhaps secondary to the impairment of cellular immunity, which is disturbed in these patients. Some reports suggest a risk 2 to 3 times higher in DM patients than in the general population (2-4). Extrapulmonary involvement and delayed diagnosis are the rule in these patients $(2,3,6,14,15)$. The clinical picture includes nonspecific symptoms such as fever and weight loss and sometimes a negative purified protein derivative tuberculin test at the time of diagnosis. The present patient had no history of TB, but a year of steroid therapy (more than $5 \mathrm{~g}$ total) combined with DM and mild renal disturbance due to diabetes nephropathy may have suppressed his cellular immunity. Although there was no pathologic evidence of TB in the false aortic aortitis, the pathological findings (the aortic wall structure was destroyed and showed necrosis) suggested an infectious. This made his condition more difficult to diagnose properly. It is important to note that mycobacteria was found as a result of a high index of suspicion.

Aneurysm is a very rare complication of TB $(11,12)$; only 42 cases of TB aneurysm of the aorta have been reported in the English literature from 1945 to the present. One early report noted that the incidence of TB aneurysm was only $0.3 \%$ (13). Although most TB aneurysms are identified by autopsy, recent reports describe successful surgical treatment of TB aneurysm when imaging can be used to determine pathogenesis. TB aneurysms rupture easily, and most are false like the one in the present case. Tubercle bacilli may reach the aortic wall in three ways (12): 1) The bacilli may implant directly on the internal surface of the vessel wall. Normally, the aortic intima is very resistant to infection. However, when this lining is altered by a condition such as atherosclerosis, resistance to infection is lowered. 2) The bacilli may be carried to adventitia or media by the vasa vasorum. 3) Involvement of the vessel wall may occur by direct infection or indirectly via the lymphatics from a contiguous focus such as a lymph node or paraspinal abscess. In the present case, it was difficult to determine which way the TB spread.

Diagnosis of miliary TB is very difficult and tends to be delayed. Therefore, it is important to investigate the possibility. The symptoms and laboratory abnormalities are nonspecific. The diagnostic clue in our case was the hypercalcemia. It is well known that hypercalcemia may complicate granulomatous disorders, such as sarcoidosis and TB (1619). In granulomatous disorders, inappropriate production of 
1,25-dihydroxyvitamin D $[1,25(\mathrm{OH} 2) \mathrm{D} 3]$ by activated macrophages and granulomas is responsible for hypercalcemia (16). Interestingly, studies have suggested that parathyroid hormone and vitamin D affect cellular immunity by affecting lymphocytes and macrophages $(18,19)$. The TB reaction is characterized morphologically by the accumulation of mononuclear cells in the subcutaneous tissue and deep and superficial dermis (19). The patient's immune status may have an important role in modulating calcium metabolism in cases of TB (17). However, one group of investigators concluded that patients with TB are not at risk for hypercalcemia either before or during treatment and they called for clarification of any actual association of hypercalcemia and TB (20). Granulomatous disease should still be considered when hypercalcemia is present, especially in an unexplainable situation like the present case with acute renal failure, which is usually associated with hypocalcemia.

Diagnosis of TB of the aorta including miliary TB can be difficult. Final diagnosis requires marrow aspiration or bone biopsy; however, aspiration was unsuccessful in our case and showed only noncaseous granuloma, a finding that does not distinguish between sarcoidosis and TB. Under suspicion of complication with sarcoidosis, we performed renal biopsy, which revealed tubulointerstitial nephritis resulting from the use of non-steroidal anti-inflammatory drugs and the ionized drug used to obtain images of the aorta. A newer diagnostic test, such as the use of DNA amplification by means of PCR of DNA fingerprinting and immunoassays for mycobacterial antigens, may provide a more rapid (a few days in comparison to a few weeks) and accurate method of identifying these fastidious organisms in the near future (21, 22). In the present case, the 4-week culture was negative. This may have occurred because of non-homogenous bone involvement or because the culture did not contain live tubercle bacilli. Bone marrow aspiration resulted in a dry tap, so we could not pursue this question further. The clinical course was highly suggestive of TB infection. Final diagnosis requires marrow aspiration or bone biopsy, however, and this patient's bone marrow showed only noncaseous granuloma, a finding that does not distinguish between sarcoidosis and TB. Renal biopsy did not reveal any granuloma. Our experience with this case underscores the importance of pursuing even the suspicion of TB. TB culture and TB PCR were both helpful in obtaining in this case.

In summary, we have presented the case of a 69-year-old man with a false abdominal aortic aneurysm associated with disseminated TB. The clue to the correct diagnosis was hypercalcemia, a paradoxical finding in a patient with acute renal failure induced by drug-based diabetic nephropathy. The final diagnosis was based on bone marrow biopsy and TB DNA PCR. TB should be considered in cases in which the patient's cellular immunity may be compromised and fever is present. Perhaps the most essential of the many things we learned from this case is the importance of examinig the data carefully, in detail; any unexplained findings should be cause for an invasive examination such as biopsy. In this case, the unexplained data provided the clue to diagnosis.

\section{References}

1. Kochi A. The global tuberculosis situation and the new control strategy of the World Health Organization. Tubercle 72: 1-6, 1991.

2. Yamada H, Katoh O, Hiura K, et al. The present status of active tuberculosis in a general hospital; a study of 186 cases. Jpn J Med 30: 304-310, 1991.

3. Shetty N, Shemko M, Vaz M, D'Souza G. An epidemiological evaluation of risk factors for tuberculosis in South India: a matched case control study. Int $\mathrm{J}$ Tuberc Lung Dis 10: 80-86, 2006.

4. Wang JY, Lee LN, Hsueh PR. Factors changing the manifestation of pulmonary tuberculosis. Int J Tuberc Lung Dis 9: 777-783, 2005.

5. Orme IM, Andersen P, Boom WH. T cell response to Mycobacterium tuberculosis. J Infect Dis 167: 1481-1491, 1993.

6. Diez M, Bleda MJ, Alcaide J, et al. Determinants of patient delay among tuberculosis cases in Spain. Eur J Public Health 14: 151155, 2004.

7. Haas DW, Des Prez RM. Mycobacterium tuberculosis. in: Principles and Practice of Infectious Disease. 4th ed. Mandell GL, Bennett JE, Dolin R, Eds. Churchill Livingstone, New York, 1995: 2213-2243.

8. Kim JH, Langston AA, Gallis HA. Miliary tuberculosis: epidemiology, clinical manifestations, diagnosis, and outcome. Rev Infect Dis 12: 583-590, 1990.

9. Proudfoot AT, Akhtar AJ, Douglas AC, Horne NW. Miliary tuberculosis in adults. Br Med J 2: 273-276, 1969.

10. Slavin RE, Walsh TJ, Pollack AD. Late generalized tuberculosis: a clinical pathologic analysis and comparison of 100 cases in the preantibiotic and antibiotic eras. Medicine Baltimore 59: 352-366, 1980.

11. Forbes TL, Harris JR, Nie RG, Lawlor DK. Tuberculous aneurysm of the supraceliac aorta - a case report. Vasc Endovasc Surg 38: 93-97, 2004.

12. Long R, Guzman R, Greenberg H, Safneck J, Hershfield E. Tuberculous mycotic aneurysm of the aorta. Review of published medical and surgical experience. Chest 115: 522-531, 1999.

13. Parkhurst GF, Decker JP. Bacterial aortitis and mycotic aneurysm of the aorta: a report of twelve cases. Am J Pathol 31: 821-835, 1955.

14. Editorial. Tuberculosis in chronic renal failure. Lancet 1: 909-910, 1980.

15. Garcia-Leoni ME, Martin-Scapa C, Rodeno $P$, Valderrabano F, Moreno S, Bouza E. High incidence of tuberculosis in renal patients. Eur J Clin Microbiol Infect Dis 9: 283-285, 1990.

16. Sharma OP. Hypercalcemia in granulomatous disorders: a clinical review. Curr Opin Pulm Med 6: 442-447, 2000.

17. Deniz O, Tozkoparan E, Yonem A, et al. Low parathormone levels and hypercalcaemia in patients with pulmonary tuberculosis: relation to radiological extent of disease and tuberculin skin test. Int $\mathbf{J}$ Tuberc Lung Dis 9: 317-321, 2005.

18. Shasha SM, Kristal B, Barzilai M, Makov UE, Shkolnik T. In vitro effect of PTH on normal T cell functions. Nephron 50: 212216, 1988.

19. Kotzmann H, Koller M, Abela C, et al. Effects of parathyroid hor- 
DOI: $10.2169 /$ internalmedicine.45.1740

mone and serum calcium on the phenotype and function of mononuclear cells in patients with primary hyperparathyroidism. Eur $\mathrm{J}$ Clin Invest 28: 353-358, 1998.

20. Kelestimur F, Guven M, Ozesmi M, Pasaoglu H. Does tuberculosis really cause hypercalcemia? J Endocrinol Invest 19: 678-681, 1996.
21. Joint Tuberculosis Committee of the British Thoracic Society. Chemotherapy and management of tuberculosis in the United Kingdom: recommendations 1998. Thorax 53: 536-548, 1998.

22. Hussein MM, Mooij JM, Roujouleh H. Tuberculosis and chronic renal disease. Semin Dial 16: 38-44, 2003.

(C) 2006 The Japanese Society of Internal Medicine http://www.naika.or.jp/imindex.html 